\title{
Actitudes hacia el español hablado y escrito de los Estados Unidos*
}

ANTONIO MEDINA RIVERA**

Recepción: 08 de febrero de 2019

Aprobación: 13 de junio de 2019

Forma de citar este artículo: Medina, A. (2019). Actitudes hacia el español hablado y escrito de los Estados Unidos. Cuadernos de Lingüística Hispánica, (34), pp 193-212.

doi

10.19053/0121053X.n34.2019.9626

* Artículo de investigación.

** PhD-University of Southern Californioa. Catedrático en Cleveland State University. Correo electrónico: a.medinarivera@csuohio. edu . (1) https://orcid.org/0000-0003-2361-6513 


\section{Resumen}

En el presente trabajo se examinan actitudes hacia el español hablado y escrito de los Estados Unidos. El análisis parte de un cuestionario que se hizo con hablantes nativos de español de diferentes países del mundo hispánico por medio de un instrumento de colección en línea que se llama Survey Monkey. En él participaron 110 hablantes de español de España, Colombia, Perú, Argentina, Cuba, Puerto Rico, Guatemala, México y Chile. Los resultados muestran tanto el conocimiento y las actitudes que dichos hablantes tienen sobre el español de los Estados Unidos, como algunos signos de vitalización y desarrollo del español en dicho país.

Palabras claves: actitudes lingüísticas, español de los Estados Unidos.

\section{Attitudes towards Spoken and Written Spanish Language of the United States}

\section{Abstract}

In this paper we analyze attitudes towards spoken and written Spanish Language of the United States. The study is based on a questionnaire submitted to native Spanish speakers from different countries of the Hispanic world through an online collection instrument called Survey Monkey. A total of 110 Spanish speakers from Spain, Colombia, Peru, Argentina, Cuba, Puerto Rico, Guatemala, México and Chile participated. The results show both the knowledge and attitudes that these speakers have about Spanish language in the United States, as well as some signs of vitalization and development of Spanish in that country.

Keywords: linguistic attitudes, Spanish language in the United States. 


\section{Attitudes vis-à-vis de l'espagnol parlé et écrit des États-Unis}

\section{Résumé}

Dans ce travail on examine des attitudes vis-à-vis de l'espagnol parlé et écrit des États-Unis. L'analyse part d'un questionnaire qui a été fait avec des natifs de l'espagnol appartenant aux différents pays du monde hispanophone. Cela a été fait avec un instrument de collecte de données en ligne qui s'appelle Survey Monkey. On a eu la participation de 110 hispanophones d'Espagne, Colombie, Pérou, Argentine, Cuba, Porto Rico, Guatemala, Mexique et Chili. Les résultats montrent tant la connaissance que les attitudes que ces gens-là ont sur l'espagnol des États-Unis, ainsi que quelques traits de revitalisation et développement de l'espagnol dans ce pays-là.

Mots clés: attitudes linguistiques, espagnol des États-Unis.

\section{Atitudes ao espanhol falado e escrito dos Estados Unidos}

\section{Resumo}

Este artigo examina atitudes em relação ao espanhol falado e escrito nos Estados Unidos. A análise é baseada em um questionário feito com falantes nativos de espanhol de diferentes países do mundo hispânico por meio de um instrumento de coleta on-line chamado Survey Monkey. Participaram 110 falantes de espanhol da Espanha, Colômbia, Peru, Argentina, Cuba, Porto Rico, Guatemala, México e Chile. Os resultados mostram os conhecimentos e atitudes que esses palestrantes têm sobre o espanhol nos Estados Unidos, bem como alguns sinais de vitalização e desenvolvimento do espanhol nesse país.

Palavras-chave: atitudes linguísticas, espanhol dos Estados Unidos. 


\section{Introducción}

El español de los Estados Unidos no se reduce a sus manifestaciones de tipo oral, sino que también cuenta con un importante desarrollo en sus registros escritos. Los medios de comunicación social, al igual que otras instituciones cívicas, educativas y religiosas, producen materiales escritos para una audiencia panhispánica de ese país. Por otro lado, hay que destacar que el español escrito de los Estados Unidos no es tan diferente al de otras variedades de español, pero, a la vez, tiene características únicas dentro de las que se incluyen la innovación léxica, la incorporación de palabras o frases del inglés y la exclusión de palabras marcadamente regionales que podrían ser problemáticas dentro de un contexto panhispánico en el que conviven diversas variedades de español. La búsqueda de un español neutro, en estos casos específicos, podría verse como una meta importante en el desarrollo del español escrito de los Estados Unidos.

En el presente estudio se examinan las actitudes y percepciones hacia el español hablado y escrito de los Estados Unidos de un grupo de hablantes que vive fuera de este entorno. El estudio incorpora un cuestionario que se distribuyó en línea a hablantes nativos de español en México, Cuba, España, Argentina, Colombia, Puerto Rico, Guatemala, Perú y Chile, el cual incluye preguntas acerca del conocimiento de los participantes sobre el español de los Estados Unidos y sobre sus percepciones del español oral y escrito de este país. Teniendo en cuenta que en los Estados Unidos reside una de las poblaciones más grandes de origen hispánico, es importante examinar las actitudes hacia una variedad de español que, sin lugar a dudas, tiene un crecimiento mundial significativo.

\section{Actitudes lingüísticas}

Janet Holmes (2013, pp. 409-411) indica algunos de los aspectos que se deben considerar en el análisis de actitudes lingüísticas:

(1.) Las actitudes hacia determinada lengua o hacia determinado dialecto son un reflejo de las actitudes que se tienen hacia esos hablantes.

(2.) La gente tiende a entender mejor aquellas lenguas o dialectos hablados por personas a las que admiran.

(3.) La gente no produce opiniones en el vacío, sino que desarrolla actitudes hacia ciertas lenguas o dialectos que reflejan sus maneras de ver o percibir a los que los hablan.

(4.) Las actitudes hacia una lengua o dialecto están influenciadas por factores políticos y sociales. 
(5.) Las actitudes lingüísticas pueden tener una gran influencia en áreas como la educación ${ }_{1}$.

Partiendo de estos criterios sería pertinente analizar la manera como los hablantes de otros dialectos de español perciben el español hablado y escrito que se usa en los Estados Unidos. Generalmente pensamos en el inglés cuando nos referimos al país, pero la realidad es que el número de hablantes de español sobrepasa al de muchos países hispanohablantes. Es interesante que Holmes (2013) mencione que las actitudes lingüísticas no se producen en el vacío, sino que reflejan las maneras de ver o percibir a los que hablan determinado dialecto (aspecto n. ${ }^{0} 3$ ). En América Latina existen opiniones diversas sobre los Estados Unidos como nación, lo cual se ve como admiración y odio a la vez. Aunque se tenga admiración por los Estados Unidos, eso no significa que también se tenga admiración por el español que ahí se habla (aspecto n. ${ }^{0}$ 2), ya que el inglés sería el idioma de referencia en este caso.

Faingold (2012) aclara que aunque el inglés no es la lengua oficial de los Estados Unidos, su estatus como lengua nacional es claro y "the U.S. constitution has not prevented the existence of implicit language policies in the United States" (p. 137). México ocupa el primer lugar en el número de hispanohablantes, y el segundo lugar se lo disputan EE.UU., Colombia, España y Argentina. Sin embargo, no todos los hablantes de español en el mundo están conscientes de la presencia y relevancia del español en los Estados Unidos. Dada la presencia del inglés como lengua dominante, en este trabajo las actitudes lingüísticas que se tienen hacia el español de los Estados Unidos podrían relacionarse con los cinco aspectos que menciona Holmes (2013), pero a la vez hay que tomar en consideración la actitud que tiene un hablante hacia una variedad con la que está en contacto y que en este caso en particular recibe influencia del inglés a diario.

El análisis de las actitudes hacia el español de los Estados Unidos puede verse como el conjunto de percepciones que se tienen de un dialecto "ad intra" (Zentella, 2007; Porcel, 2012; Medina-Rivera, 2014). En este estudio en particular nos interesa examinar las opiniones "ad extra," o sea las opiniones o percepciones que se tienen del español de los Estados Unidos fuera del país. Este tipo de evaluación puede relacionarse con el conocimiento que la gente de otros países de habla hispana tiene sobre el español oral y escrito de los Estados Unidos. También hay que tener en cuenta que los juicios sobre un dialecto o lengua suelen referirse a la manera como percibimos el lenguaje oral y no necesariamente el escrito.

1 Actitudes lingüísticas (pág 198) aparece 5 enumeraciones, la cita corresponde a la 5 númeración. 
En resumen, este trabajo tiene como finalidad destacar el conocimiento que hay sobre el español y las actitudes hacia dicha lengua por parte de hablantes de español que viven fuera de los Estados Unidos. Para el análisis de las actitudes lingüísticas se tomarán en cuenta los criterios presentados por Holmes (2013) - sobre todo la actitud que hay hacia la gente de determinado país -, pero también se considerará el efecto que tiene la mezcla y la influencia que se da como resultado del contacto de ambas lenguas.

\section{Metodología}

En este estudio se incorporó un cuestionario de actitudes lingüísticas (ver Apéndice) distribuido por medio de Survey Monkey, un instrumento en línea que facilita la recolección de datos de una manera rápida, efectiva y con carácter anónimo. El cuestionario consta de 14 preguntas: la primera parte incluye información demográfica y la segunda incluye preguntas que tienen que ver con actitudes, percepciones y conocimiento de los participantes. Se seleccionaron participantes de diez países de habla hispana. Los requisitos mínimos para participar en el cuestionario eran que los participantes fueran mayores de 18 años, que estuvieran residiendo en su país de origen y que no hubieran residido en los Estados Unidos. Para poder reclutar participantes y mantener su anonimato se contó con la ayuda de personas que sirvieron de contacto en cada uno de los países seleccionados para el estudio. Cada contacto se encargó de enviar el enlace del cuestionario en línea a un número de personas de su país y así el investigador principal no tuvo que tener ningún tipo de conexión con los participantes.

Se trató de cubrir zonas diferentes del mundo hispánico para este estudio (España, América del Norte, América del Sur y el Caribe). Survey Monkey tiene la opción de servir como un cuestionario abierto y la administración del servicio se encarga de enviarlo a todos sus usuarios, pero también ofrece la oportunidad de mantenerse como cuestionario cerrado o discreto. En este caso se optó por la segunda opción. Dada las características específicas que se necesitaban para los participantes de este estudio, se les dieron instrucciones claras y específicas a los contactos de cada país y se aclararon dudas al respecto cuando era necesario. A este tipo de muestra se le conoce como "judgmental sampling", ya que se requiere de un cuidado especial en la selección de los participantes. Este tipo de muestra

is used in cases where the specialty of an authority can select a more representative sample that can bring more accurate results than by using other probability sampling techniques. The process involves nothing but purposely handpicking 
individuals from the population based on the authority's or the researcher's knowledge and judgment. (Explorable.com, 2009)

\subsection{Participantes}

En el estudio se incluyó un total de 110 participantes, todos de clase media 0 media alta (estudiantes universitarios y profesionales). Se eliminaron los cuestionarios incompletos y aquellos cuestionarios consecutivos (que entraron al sistema casi simultáneamente) que contenían respuestas idénticas. Este tipo de cuestionarios con respuestas idénticas puede ocurrir cuando el usuario oprime el botón de "enviar" más de una vez y como los cuestionarios eran anónimos no había otra manera de identificarlos o distinguirlos, así que se optó por eliminarlos para no sacrificar la integridad del estudio. Se hizo uso de SPSS (incluyendo tabulación cruzada) para el análisis estadístico de los resultados. También se limitó el número máximo de participantes por cada país a 15 para que no hubiera tanta disparidad. En los casos en los que se recibieron más de 15 cuestionarios de un país determinado, simplemente se optó por los primeros 15 que entraron al sistema y que cumplían con los requisitos mínimos. La Tabla 1 muestra el número de participantes por país:

Tabla 1. Participantes

\begin{tabular}{cc}
\hline México & 10 \\
Puerto Rico & 11 \\
España & 14 \\
Colombia & 13 \\
Perú & 12 \\
Cuba & 2 \\
Guatemala & 10 \\
Argentina & 12 \\
Chile & 15 \\
Venezuela & 11 \\
\hline
\end{tabular}

\section{Resultados}

En el apéndice de este trabajo se incluye una copia del cuestionario que se utilizó para este estudio. De acuerdo con las respuestas del estudio, el 53.64\% de los participan- 
tes habla otra lengua aparte del español. Las lenguas más comunes que se mencionaron fueron inglés, francés, italiano y gallego (nuestro contacto de España provenía de Galicia).

De los diez países seleccionados para este estudio, Puerto Rico tiene el inglés como lengua oficial con el español. Dada la condición colonial del país, la cual se remonta a 1898, muchos puertorriqueños tienen algún tipo de conexión estrecha con los Estados Unidos; sin embargo, eso no implica que tengan un conocimiento claro sobre el número de hablantes de español que viven en los Estados Unidos o sobre la realidad de dicho idioma en el país.

En el caso particular de España, la Oficina Estadística Comunitaria Eurostat indica que este es el tercer país de la Unión Europea, detrás de Hungría y Portugal, con un porcentaje mayor de adultos que no habla una lengua extranjera (46.6\%). En la América Latina hispana, el escenario lingüístico podría ser aun más complejo que el de España, ya que existen muchas lenguas indígenas en la zona y personas que no hablan español 0 lo hablan con dominio moderado. De acuerdo con English Proficiency Index, Argentina es el líder, entre los otros países latinoamericanos, con respecto al nivel de aprendizaje y adquisición del inglés como segunda lengua.

En cuanto a la edad de los participantes, el $84 \%$ tenía entre 18 a 25 años, y el 26 tenía más de 25. Esta distribución etaria no parece sorprendente, ya que al ser un cuestionario en línea, se espera que los más jóvenes tengan una mayor disposición a participar. A la vez, como los participantes eran más jóvenes, se esperaba que tuvieran un conocimiento y un contacto con los Estados Unidos mucho más limitado que los más adultos. Esta suposición se basa en la idea de que los adultos han tenido mayores oportunidades para viajar y para haberse expuesto a diversos elementos de la cultura estadounidense. La realidad es que los resultados no mostraron ninguna relación significativa al respecto, partiendo de las respuestas a la pregunta $n .^{0} 11$ del cuestionario en tabulación cruzada con la edad de los participantes.

La distribución de los participantes por género fue de $84 \%$ de participantes del género femenino y $26 \%$ del masculino. Tampoco se pudo establecer una relación estadísticamente significativa con el género de los participantes. Como se acaba de observar, las primeras cuatro preguntas eran de contenido demográfico. William Smith ofrece sus observaciones sobre la participación del público general en cuestionarios:

Survey response and non-response studies have shown that trends in who responds to surveys do indeed exist, at least with regard to traditional modes of survey administration. In general, more educated and more affluent people are more 
likely to participate in surveys than less educated and less affluent people (Curtin, Presser, and Singer, 2000; Goyder, Warriner, \& Miller, 2002; Singer, van Hoewyk, \& Maher, 2000), women are more likely to participate than men (Curtin et al. 2000; Moore \& Tarnai, 2002; Singer et al. 2000), younger people are more likely to participate than older people (Goyder, 1986; Moore \& Tarnai, 2002), and white people are more likely to participate than non-white people (Curtin et al. 2000; Groves, Singer, \& Corning, 2000; Voigt, Koepsell \& Daling, 2003). (Smith, 2008, p. 3)

La participación en el presente estudio muestra algunas de las tendencias que menciona Smith (2008) en su investigación: nivel educativo más alto, mujeres y jóvenes como los que participan con mayor frecuencia. Aunque en el cuestionario no se les preguntó el nivel educativo a los participantes, este se deriva del nivel educativo y social de nuestros contactos (clase media o media alta, con nivel universitario) y el acceso de los participantes a computadora y servicio de internet.

\subsection{Actitudes lingüísticas}

Las próximas preguntas del cuestionario se asocian a actitudes, percepciones y conocimiento de los participantes sobre el español hablado y escrito de los Estados Unidos. La pregunta n. ${ }^{\circ}$, la cual hace referencia a los fundamentos 1 y 2 de la definición de Holmes (2013) que se presentó anteriormente, ya que pide a los participantes que comparen el español de los Estrados Unidos con el de otras variedades del mundo hispánico. Los resultados se muestran en la Tabla 2:

Tabla 2. El español hablado de los Estados Unidos en comparación con otras variedades

\begin{tabular}{ll}
\hline Comparable al de otras variedades de español & $23.42 \%$ \\
Diferente, con sus rasgos propios & $36.94 \%$ \\
Deficiente, contaminado de anglicismos & $39.64 \%$ \\
\hline
\end{tabular}

Las primeras dos alternativas corresponden a respuestas positivas o neutrales, mientras que la tercera evidencia una opinión negativa. Solamente el $23.42 \%$ de los participantes opina que el español hablado de los Estados Unidos es comparable con el de otras variedades del mundo hispánico. Aunque la tabulación cruzada entre el país de origen del participante y la actitud hacia el español oral no resultó significativa (Chi-cuadrada $=.095, \mathrm{p}>.05$ ), cabe mencionar que los participantes de Colombia mostraron los niveles más altos de respuestas negativas (10 en total), y los participantes de España obtuvieron el porcentaje mayor de respuestas positivas con 6. 
La pregunta n. ${ }^{\circ} 6$ es similar a la n. ${ }^{\circ} 5$, pero esta vez haciendo referencia al español escrito, tal como se ve en la Tabla 3:

Tabla 3. El español escrito de los Estados Unidos en comparación con otras variedades

\begin{tabular}{ll}
\hline Comparable al de otras variedades de español & $31.48 \%$ \\
Diferente, con sus rasgos propios & $36.11 \%$ \\
Deficiente, contaminado de anglicismos & $32.41 \%$ \\
$\mathrm{n}=108^{2}$ & \\
\hline
\end{tabular}

Resulta interesante que, en conjunto, las actitudes hacia el español escrito obtuvieron evaluaciones más positivas o neutrales. Las evaluaciones hacia el español escrito resultan más positivas, dado la clara distinción que muchos hablantes tienen entre los registros hablados y escritos, y perciben la escritura como un registro más cuidadoso en su producción, tal como se ve en el continuo de registros de Finegan y Biber (1994). En su estudio, estos autores (1994) establecieron un modelo para el análisis de variación de registros, en el cual se incorporaron tres parámetros situacionales: oportunidad para la producción cuidadosa, propósitos de la comunicación y grado o nivel de contexto compartido (p. 321). En dicho modelo se incluyen siete registros diferentes para examinar diferencias dentro de un continuo lingüístico: cartas personales, ficción en general, reportaje periodístico, prosa académica, conversaciones, entrevistas y discursos públicos.

Generalmente, la evaluación que la gente hace de una lengua o de un dialecto de un grupo determinado tiene como referencia la lengua hablada y no la escrita. En términos gramaticales, las diferencias que se perciben entre las diferentes variedades de español (al igual que en otras lenguas) son más evidentes en la lengua oral y en este sentido el español escrito tiende a ser bastante homogéneo o estandarizado. La correlación entre el país de origen de los participantes y la actitud hacia el español escrito resultó significativa (Chi-cuadrada $=.037, \mathrm{p}<.05$ ). Los participantes de España y Perú (8 respuestas vs. 6 respuestas, respectivamente) mostraron el porcentaje mayor de respuestas positivas hacia el español escrito de los Estados Unidos, mientras que los participantes de Colombia (8 en total) mostraron el porcentaje más alto de respuestas negativas. Con respecto a respuestas neutrales, los participantes de Chile y Puerto Rico mostraron el número mayor de respuestas con 9 y 6 , respectivamente.

2 Hay que recordar que no todos los participantes responden a todas las preguntas de un cuestionario, por eso el valor de n varía de 108 a 110. 
La pregunta n. $^{0} 6$ tiene también como objetivo destacar la importancia del español escrito y el conocimiento que se tiene sobre el mismo. Holmes (2013, pp. 409- señala en su definición que la gente no produce opiniones en el vacío, sino que desarrolla actitudes hacia ciertas lenguas o dialectos que reflejan su manera de ver o percibir a los que los hablan dichas lenguas o dialectos; pero a esto hay que añadir que las actitudes lingüísticas también son el resultado de la falta de conocimiento sobre una variedad o dialecto. Mayor-Marsán, en su artículo del Centro Virtual Cervantes (2008), provee ejemplos sobre los esfuerzos de producir materiales en español dentro del contexto social y cultural de los Estados Unidos. El autor (2008) señala que "Hasta hace muy poco tiempo „el 90\% de los libros en español en los Estados Unidos eran publicados en el extranjero", de acuerdo con lo declarado por Lisa Alpert, editora del sello Random House Español, a la cadena noticiosa CNN en español en el 2001" (p. 915). En su artículo, Mayor Marsán ofrece una lista bastante completa de las casas publicadoras que producen libros en español en los Estados Unidos. A esa lista habría que añadir instituciones religiosas como St. Mary"s Press, Liguori Publications y United States Catholic Conference of Bishops, las cuales producen libros y materiales en español para una audiencia panhispánica. Partiendo de mi propia experiencia con estas instituciones, puedo constatar que St. Mary"s Press y la United States Catholic Conference cuentan con guías para la redacción en español, con un énfasis especial de cómo escribir para una audiencia panhispánica/multidialectal en los Estados Unidos. Estas guías proveen ejemplos de palabras y expresiones que son comúnmente usadas en el ambiente pastoral del apostolado hispano.

La pregunta $\mathrm{n}^{0} \mathrm{7}$ se refiere al conocimiento de los participantes sobre la presencia de la Real Academia Española en los Estados Unidos. Solamente un 42.56 \% dijo tener conocimiento sobre la existencia de la misma. En realidad, la Academia Norteamericana de la Lengua es la más joven entre las 22 academias del español en el mundo. Se fundó en 1973 y quedó incorporada oficialmente en 1980. La fundación de esta academia es una manera de reconocer la presencia e importancia del español en los Estados Unidos; sin embargo, muchas personas en los Estados Unidos tampoco saben de la existencia de dicha institución y del trabajo que realiza para la preservación del idioma en el país.

En la pregunta $n .^{0} 8$ se averiguó a los participantes si sabían cuál era la lengua que más se habla en los Estados Unidos además del inglés y el 90.09 \% pudo identificar al español. Otras respuestas incluyeron el chino (5.41\%) y el francés (4.50\%). Las noticias internacionales sobre el tema de inmigración, las olas migratorias provenientes de países con conflictos políticos o sociales como México, Cuba, El Salvador, Colombia, etc., y los bombardeos de información que muchos reciben por medio del internet y las redes sociales pueden también contribuir al conocimiento que se tiene sobre los Estados 
Unidos (ya sea cierto, tergiversado o completamente equivocado). De acuerdo con el US Census Bureau (2016), hay alrededor de 54 millones de personas de origen hispano en los Estados Unidos, lo que equivale al 17 \% de la población total. Solamente México, con 127 millones de habitantes, tiene el mayor número de personas de origen hispano en el mundo. La población de Colombia se estima en los 48.2 millones, la de España en 46.4 millones, y la de Argentina en 42.4 millones (Population Reference Bureau, 2015).

La Oficina del Censo indica que unos 41 millones de personas en los Estados Unidos pueden hablar español como primera lengua (lengua dominante) y otros 11.6 millones pueden hablarlo como segunda lengua. En la pregunta n. ${ }^{0} 10$, los participantes identificaron a México, España, Argentina, Colombia y los Estados Unidos como los cinco países con mayor número de hispanos en el mundo. Otras respuestas incluyeron a Venezuela y al Perú. Aunque el orden no resultara correcto con los números que ofrece el World Population Data Sheet 0 el Population Reference Review, al menos los participantes mostraron conocimiento sobre el tema y parecen estar al tanto de la importancia de los Estados Unidos como un país con una fuerte presencia hispánica.

Continuando con las preguntas del cuestionario, la n. ${ }^{0} 11$ tiene como objetivo determinar el conocimiento que posee el hablante sobre el uso y la difusión del español de los Estados Unidos e incluye una serie de aseveraciones sobre la radio, la televisión, los periódicos y las casas publicadoras. Se les pidió a los participantes que escogieran aquellas aseveraciones que consideraban ciertas. En la Tabla 4 se presentan los resultados:

Tabla 4. Radio, televisión, periódicos, publicaciones y la presencia del español en los Estados Unidos

$\begin{array}{llr}\text { 1. } & \text { En EE. UU. hay algunas estaciones de radio en español (menos de 50)3 } & 63.96 \% \\ \text { 2. } & \text { En EE. UU. hay muchas estaciones de radio en español (más de 50) } & 29.36 \% \\ \text { 3. } & \text { En EE. UU. hay un solo canal televisivo en español } & 6.31 \% \\ \text { 4. } & \text { En EE. UU. hay varios canales televisivos en español } & 86.49 \% \\ \text { 5. } & \text { En EE. UU. hay un número muy limitado de periódicos y revistas en español } & 49.55 \% \\ \text { 6. } & \text { En EE. UU. hay muchos de periódicos y revistas en español } & 39.64 \% \\ \text { 7. } & \text { EE. UU. es un buen lugar para aprender español } & 17.12 \% \\ \text { 8. } & \text { EE. UU. es un buen lugar para estudiar literatura/lingüística hispánica } & 35.14 \% \\ \text { 9. Hay escritores literarios que escriben en español en EE. UU. } & 60.36 \% \\ \text { 10. } & \text { El español en EE. UU. está en aumento } & \end{array}$

3 Debido a que los términos "algunos" y "muchos" son muy relativos, optamos por usar el número 50 como referencia. 
Cada una de las aseveraciones anteriores podría ser parte de una discusión detallada 0 el tema para una investigación, por lo tanto, solamente se harán algunas observaciones. Las oraciones 10, 4, 1 y 9 obtuvieron los porcentajes más altos. Parece ser de conocimiento general que la presencia del español en los Estados Unidos ha ido en aumento durante los últimos años, y que ese aumento y relevancia se refleja en el número de estaciones de radio y televisión. De acuerdo con el Latin Music Wire había un total de 10000 estaciones de radio comerciales y 2500 estaciones no comerciales en el 2011 (Latin Music Wire, 2011). Solamente en español había 495 estaciones comerciales, lo que equivale al $5 \%$ del número total.

El crecimiento e impacto de la televisión en español también parece ser muy relevante. En muchos hogares de los Estados Unidos la televisión en español forma parte del diario vivir. Visto de esa manera, la televisión en español tendría un impacto significativo en el mantenimiento del español en los Estados Unidos. Para los hablantes de español como segunda lengua, la televisión en español se convierte en una oportunidad para mejorar sus destrezas lingüísticas. Dentro de la programación televisiva hay programas exportados de otros países de habla hispana como México y Colombia, pero a la vez hay otros programas (e.g. noticias, programas de entretenimiento) que se producen en los Estados Unidos. Según Albarran y Hutton (2009, p. 14), los hispanos dedican más horas a escuchar radio y ver televisión, comparados con otros grupos étnicos, y las compañías comerciales aprovechan la oportunidad de mercadearse y promocionar sus productos. En la actualidad, los comerciales en español no solamente se anuncian en las cadenas hispanas, sino también en eventos importantes de otras cadenas, como el Super Bowl, los premios Grammy y otros programas de entretenimiento.

Además de los libros y las revistas académicas que se publican en español en los Estados Unidos, hay que mencionar los periódicos y las revistas populares. Muchas ciudades grandes de los Estados Unidos cuentan con uno o dos periódicos en español, y varias revistas como Cosmopolitan y Men's Health las producen también en versión española para los Estados Unidos y para el mercado latinoamericano. El artículo de Mayor-Marsán (2008) reconoce el crecimiento que han tenido las publicaciones en español durante los últimos 20 años, y a eso también hay que añadir el desarrollo de materiales religiosos en español para una audiencia panhispánica.

La oración n. ${ }^{0} 7$ presenta la idea de percibir a los Estados Unidos como un lugar aceptable en el que una persona podría aprender y adquirir el español. El porcentaje de $17.2 \%$ no parece sorprendente, a pesar de que el español es la "lengua extranjera". Sin lugar a dudas, una persona puede aprender a hablar español sin tener que salir de los 
Estados Unidos y adquirir un nivel de dominio alto (Medina-Rivera, 2004). La oración n. ${ }^{0}$ 8 ofrece un panorama similar al de la n. ${ }^{0} 7$, al señalar a los Estados Unidos como un lugar en el que un estudiante puede obtener un título en literatura o lingüística en español. En este caso, el porcentaje de la aseveración n.$^{0} 8$ resultó mayor $(35.14 \%$ ), quizás por la reputación internacional que tienen las universidades estadounidenses en el mundo, y porque nuestros contactos de cada país son personas que han obtenido títulos universitarios en los Estados Unidos.

Resulta interesante que la oración n. ${ }^{0} 9$ mostrara un porcentaje de 60.36 \% (uno de los más altos), ya que autores de talla internacional como Isabel Allende, Carlos Ruiz Zafón y Daína Chaviano viven en los Estados Unidos y publican sus libros en español en casas publicadoras de los Estados Unidos. Entre esas casas podríamos mencionar a Vintage Español, la cual se ha convertido en una casa publicadora de gran importancia y alcance mundial.

\subsection{Conexiones y contactos con los Estados Unidos}

Las últimas tres preguntas del cuestionario tienen que ver con el nivel de familiaridad que tienen los participantes con los Estados Unidos y sus actitudes generales con respecto al país. Nuevamente se hace eco a lo que establece Holmes (2013) cuando señala que las actitudes hacia determinada lengua o dialecto son un reflejo de las actitudes que se tienen hacia sus hablantes (pp. 409-411). En las preguntas 12 y 13 se les averigua a los participantes si han visitado los Estados Unidos y si tienen familiares o amigos que vivan ahí. Tal como se indicó anteriormente, en la selección de los participantes se requería que estos no hubieran vivido en los Estados Unidos. El $53.15 \%$ respondió que había visitado ese país, y el 81.08 \% que tenía familiares o amigos allí. Aunque no se obtuvo ninguna relación significativa con estos factores, podría sugerirse que el haber estado expuesto directamente a la cultura y vida de los Estados Unidos podría tener un efecto en las actitudes que se tienen hacia el país. Salter y Teger (1975) sugieren que en términos de contacto con otros países o culturas, "if the contact was generally satisfying, attitudes were enhanced by a generalization of the consequent glow of positive feelings. If the contact was relatively unpleasant, then these negative feelings generalized to attitudes, and thus reduced their favorableness" (p. 221). Este comentario de Salter y Teger puede relacionarse directamente con las observaciones de Holmes (2013). En este sentido, las actitudes hacia un país o cultura tienen su base en las experiencias (positivas o negativas) de cada individuo. 
La última pregunta sirve para que cada uno de los participantes exprese sus sentimientos hacia los Estados Unidos de una manera directa. La Tabla 5 muestra los resultados:

Tabla 5. Actitudes hacia los Estados Unidos

\begin{tabular}{lr}
\hline Favorable & $51.82 \%$ \\
Neutral & $40.0 \%$ \\
No favorable & $8.18 \%$ \\
\hline
\end{tabular}

Solamente el $8.18 \%$ de los encuestados mostró una actitud negativa hacia los Estados Unidos. En el caso particular de este estudio, las actitudes negativas no son hacia el país en general, sino al uso del español oral y escrito, tal como se vio en las tablas 2 y 3. Podría sugerirse además que el haber visitado el país y el tener familiares y amigos que viven en él, podría ayudar a tener una actitud más positiva hacia los Estados Unidos. Nuevamente no se encontró una relación estadísticamente significativa entre estos factores y cabe señalar que, según Holmes, "Attitudes to language are strongly influenced by social and political factors" (2013, p. 410). Sin lugar a dudas, las actitudes hacia un país pueden variar rápidamente de un periodo a otro, con un cambio de gobierno o con el cambio en las relaciones entre dos países.

\section{Conclusión}

El objetivo principal de este trabajo era destacar y examinar el conocimiento que tienen los hablantes de otros países hispanohablantes sobre el español oral y escrito de los Estados Unidos, e identificar las actitudes y percepciones de estos hablantes hacia el español de los Estados Unidos. Resultó evidente que los participantes del estudio cuentan con un conocimiento básico sobre la presencia del español en los Estados Unidos. Aunque no se percibió una actitud abrumadoramente negativa hacia el español oral y escrito de los Estados Unidos, tampoco puede decirse que se percibe como una variedad en relación de igualdad con otras variedades del español. Para poder ofrecer un panorama más completo sobre la realidad del español escrito, se hizo referencia al constante crecimiento en la producción de libros y otros materiales escritos en español dirigida a los latinos de los Estados Unidos. El hecho de que haya una producción de materiales en español para una audiencia panhispánica puede llevarnos al desarrollo y existencia de una norma culta del español en los Estados Unidos, como se da en los demás países hispanohablantes. Asimismo, al resaltar la importancia del español escrito se abre espacio 
para futuras investigaciones sobre el tema y el efecto que podría tener el español escrito en el mantenimiento y mayor vitalidad del español en los Estados Unidos.

Los datos que se obtuvieron en este estudio son una muestra de la manera de llegar a una audiencia fuera de los Estados Unidos para examinar las actitudes que se tienen hacia el español de este país. La incorporación de Survey Monkey resultó ser una manera efectiva y rápida para llegar a los participantes de este estudio de una manera anónima. Un total de 110 personas de habla hispana participaron en el cuestionario, y aunque podría parecer un número relativamente limitado, sirvió para obtener las evaluaciones de los participantes de otros países.

El desarrollo y el crecimiento del español oral y escrito de los Estados Unidos nos ayudan a tener una mayor comprensión del estatus de esta lengua en el ámbito internacional. El estudio de las actitudes lingüísticas es un componente importante para

entender las opiniones y percepciones sobre una lengua o dialecto. En el futuro se puede expandir el tema acerca de las actitudes que tienen los habitantes de otros países de habla hispana hacia el español de los Estados Unidos, pero también hay que poner mayor atención al hecho de que el español de los Estados Unidos se enfrenta a una realidad multidialectal o panhispánica que lo hace diferente al español de otros países del mundo hispánico.

\section{Apéndice}

\section{Cuestionario sobre el español de los Estados Unidos Información demográfica:}

1. País de origen:

2. Lenguas que hablas:

3. Edad: menor de 25 años mayor de 25 años

4. Género: Masculino Femenino

5. En términos generales, el español hablado de los Estados Unidos:

a. Es comparable al español de otros países de habla hispana.

b. Es un español diferente, con sus propias características.

c. Es un español deficiente y contaminado de anglicismos.

6. En términos generales, el español escrito de los Estados Unidos: 
a. Es comparable al español de otros países de habla hispana.

b. Es un español diferente, con sus propias características.

c. Es un español deficiente y contaminado de anglicismos.

7. En los Estados Unidos:

a. Existe una Academia de la Lengua Española.

b. No existe una Academia de la Lengua Española.

8. En los Estados Unidos:

a. Existen casas editoriales que producen libros en español.

b. No existen casas editoriales que producen libros en español.

9. Después del inglés, el segundo idioma más hablado en los Estados Unidos es:

a. Alemán

b. Chino

d. Español

d. Francés

10. Según tu conocimiento, los cuatro países con mayor número de hispanohablantes son:

11. Marca con una $X$, aquellas aseveraciones que según tu conocimiento son ciertas:

En los Estados Unidos hay muchas estaciones de radio en español (más de 50)

En los Estados Unidos hay algunas estaciones de radio en español (menos de 50)

En los Estados Unidos hay un solo canal televisivo en español

En los Estados Unidos hay varios canales televisivos en español

En los Estados Unidos hay un número muy limitado de periódicos y revistas en

español 
En los Estados Unidos hay muchos periódicos y revistas en español Los Estados Unidos es un buen lugar para aprender español Los Estados Unidos es un buen lugar para estudiar literatura/lingüística hispánica a nivel universitario

Hay escritores literarios que escriben en español en los Estados Unidos El español en los Estados Unidos está en aumento

12. ¿Has visitado o vivido en los Estados Unidos?

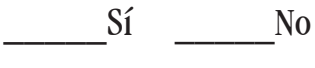

Explica:

13. ¿Tienes familiares o amigos que viven en los Estados Unidos?

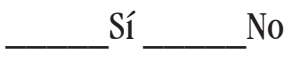

Explica:

14. ¿Cómo describirías tu actitud o percepción de los Estados Unidos en términos generales?

Favorable

No favorable

Neutral

\section{Referencias}

Albarran, A. B. \& Hutton, B. (2009). A History of Spanish Language Radio in the United

States. Denton, TX: Center for Spanish Language Media.

Explorable.com (2009). Judgmental Sampling. Retrieved from https://explorable.com/ judgmental-sampling 
Faingold, E. (2012). Official English in the Constitutions and Statutes of the Fifty States in the United States. Language Problems and Language Planning, 36(2), 136-148.

Finegan, E. \& Biber, D. (1994). Register and Social Dialect Variation: An Integrated Approach. In D. Biber \& E. Finegan (Eds.), Sociolinguistic Perspectives on Register (pp. 315-347). Oxford, England, New York, NY: Oxford University Press.

Holmes, J. (2013). An Introduction to Sociolinguistics (4th ed.), London, England:

Routledge.

Latin Music Wire. (2011). El número de estaciones de radio en los Estados Unidos. Recuperado de http://www.latinmusicwire.com/home/el-numero-de-estaciones-de-radio-en-losestados-unidos

Mayor-Marsán, M. (2008). Editoriales que se dedican a la publicación de libros en español en los Estados Unidos. En Enciclopedia del español de EE.UU. (Centro

Virtual Cervantes). Madrid, España: Instituto Cervantes.

Medina-Rivera, A. (2014). Actitudes lingüísticas entre hablantes nativos hacia las variedades de español en el área metropolitana de Cleveland. Revista Internacional de Lingüística Aplicada, 12(1), 61-76.

Population Reference Bureau (2015). World Population Data Sheet 2015. Retrieved from https:// www.prb.org/world-population-2015/

Porcel, J. (2012). Language Maintenance and Language Shift among US Latinos. In M.

Díaz-Campos (Ed.), The Handbook of Hispanic Sociolinguistics (pp. 623-645).

Malden, MA: Wiley-Blackwell.

Salter, C. A. \& Teger, A. I. (1975). Change in Attitudes toward other Nations as a Function of the Type of International Contact. Sociometry, 38(2), 213-22.

Smith, W. (2008). Does Gender Influence Online Survey Participation?: A Record-Linkage Analysis of University Faculty Online Survey Response Behavior. Retrieved from http://files.eric. ed.gov/fulltext/ED501717.pdf

United States Census Bureau (2016, June 23). Only County in Nation With Majority of

Population Age 65 or Older. Retrieved from https://www.census.gov/newsroom/pressreleases/2016/cb16-107.html 
Zentella, A. C. (2007). "Dime con quién hablas, y te diré quién eres": Linguistic (in)security and Latina/o unity. In J. Flores \& R. Rosaldo (Eds). The Blackwell Companion to Latino Studies (pp. 25-39). Malden, MA: Blackwell. 